\title{
High accuracy of a new robotically assisted technique for total knee arthroplasty: an in vivo study
}

\author{
Stefano Marco Paolo Rossi ${ }^{1}\left[\right.$ [ Rudy Sangaletti ${ }^{1,3} \cdot$ Loris Perticarini $^{1} \cdot$ Flavio Terragnoli $^{2} \cdot$ Francesco Benazzo $^{1,3}$
}

Received: 3 July 2021 / Accepted: 4 November 2021 / Published online: 4 January 2022

(c) The Author(s) under exclusive licence to European Society of Sports Traumatology, Knee Surgery, Arthroscopy (ESSKA) 2021

\begin{abstract}
Purpose Over the last decade, robotic TKA gained popularity for improving the accuracy of implant positioning and reducing outliers in limb alignment comparing to conventional jig-based TKA. Hypothesis of this study was that this newly designed robotically assisted system will achieve a high level of accuracy for bone resection. Purpose of the study was to evaluate the accuracy of the system.

Methods For this study, 75 knees in 75 patients were operated using a new, robotic system $\left(\right.$ ROSA $^{\circledR}$ Knee System; Zimmer Biomet, Warsaw, IN) with a Posterior Stabilized Total Knee Arthroplasty (Persona ${ }^{\circledR}$ Knee System). The planned, validated and measured angles and cuts for the distal and posterior femur, for the proximal tibia and for the final coronal alignment on long standing x-rays were compared.

Results A statistically significant difference was found only between the average planned and the average validated angle for femoral flexion, tibial coronal axis, medial and lateral cuts; the average difference was in any case below $1 \mathrm{~mm}$ or under 1 degree with $\mathrm{SD}<1$. No statistical difference was found between planned validated and measured cuts. Average difference between planned HKA and measured was $1.2 \pm 1.1$. No statistically significant difference was found.

Conclusions The results of this study demonstrated that using this new surgical robot in total knee arthroplasty it is possible to perform accurate bone cuts and to achieve the planned angles and resections.
\end{abstract}

Keywords Robotic surgery · Total knee arthroplasty · Navigation · Computer assisted surgery $\cdot$ In vivo · Accuracy

Stefano Marco Paolo Rossi

rossi.smp@gmail.com

1 Sezione di Chirurgia Protesica ad Indirizzo Robotico,

Unità di Traumatologia dello Sport, U.O.C. Ortopedia e

Traumatologia Fondazione Poliambulanza, Via Bissolati, 57,

25124 Brescia, Italy

2 U.O.C. Ortopedia e Traumatologia, Fondazione Poliambulanza, Via Bissolati 57, 25124 Brescia, Italy

3 Università degli Studi di Pavia, Pavia, Italy 


\section{Introduction}

Different authors investigated the outcome of total knee arthroplasty (TKA) and demonstrated that up to $20 \%$ of the patients are not satisfied with their knee function after TKA $[4,9,10,13,20]$ Pain, instability, and limitation of the range of motion are the most commonly reported symptoms among unsatisfied patients, while the major causes reported of unsuccessful TKA are inadequate alignment and errors in balancing $[4,9]$. With the aim of improving outcomes, there is an increasing focus on different alignment options [17] and soft-tissue balancing techniques [18]. As standard instrumentations might not be accurate enough to achieve either of these, balancing tools, patient-specific instrumentations (PSI) and, more recently, computer and robotic-assisted TKA have been introduced to help surgeons to adopt hybrid techniques and, at the same time, to optimize alignment, reducing the number of outliers $[6,13,14,21]$.

Computer navigation provides patient-specific anatomical data with recommendations for bone resection and optimal implant positioning, but the computer system does not actively control or restrain the motor skill of the operating surgeon. Over the last decade, robotic TKA gained popularity for improving the accuracy of implant positioning and reducing outliers in limb alignment comparing to conventional jig-based TKA $[13,14]$. Robotic tools are classified in fully active versus semi-active systems [5]. Fully active robotic systems work autonomously to perform the planned femoral and tibial bone resections. Semi-active robotic systems enable the surgeon to maintain the overall control over bone resections and implant positioning, but provide live intraoperative feedback to limit deviation from the preoperative and intraoperative surgical plan [5]. The Rosa ${ }^{\circledR}$ (Zimmer-Biomet Warsaw IN) robotic-arm system is a new robotic system that incorporates robotic placement of the cutting jigs and dynamic ligament balance evaluation. This system can be considered as a collaborative robotic system with the robot helping in placing the cutting jig, while the surgeon is in full control of sawing through the jig [19].

The aim of this study was to analyse the accuracy of this new system to perform the planned bone cuts and angles in an imageless procedure. The primary hypothesis was that the robotic system was reliable in terms of accuracy of planning, execution and validation of the bone cuts and angles. To demonstrate this hypothesis, the planned validated and measured bone cuts and angles were compared. This is, to our knowledge, the first validation study performed in vivo with the Rosa ${ }^{\circledR}$ system.

\section{Materials and methods}

From March 2020 to December 2020, 85 consecutive patients requiring TKA to treat primary osteoarthritis were enrolled in a prospective study; the first 10 cases were excluded as included in the learning curve period [9, 10], so the data of 75 procedures are presented. Mean age at the time of surgery was $70.6( \pm 12)$ years with a mean body mass index (BMI) of $29 \mathrm{~kg} / \mathrm{m}^{2}( \pm 14), 37$ were females. All surgeries were performed by the same surgical team (including the two senior authors and one younger author, all experienced in computer assisted knee surgery). In all patients the same implant was used (Persona PS, Zimmer Biomet, Warsaw, IN). All the procedures were performed with the assistance of ROSA Knee System (Zimmer Biomet, Warsaw, IN) using the imageless option. Planning was generated from landmarks taken on the femur and tibia after arthrotomy. Landmarks are standard landmarks for navigation systems and include centre of the femoral head, femoral canal entry point, trochlear groove, anterior cortex, epicondyles, distal and posterior femoral condyles, malleoli, medial and lateral tibial plate, tibial canal entry, cruciate ligaments' insertions and tibial tuberosity. An intraoperative plan was defined and validated according to the surgeon's preference as for the final alignment decided, and then, bone resections were performed with the assistance of the robot. All the cuts were verified by the tools offered by the Rosa system and, for each bone cut, the resection thickness was measured using a dedicated Vernier calliper with a $0.5 \mathrm{~mm}$ accuracy as described

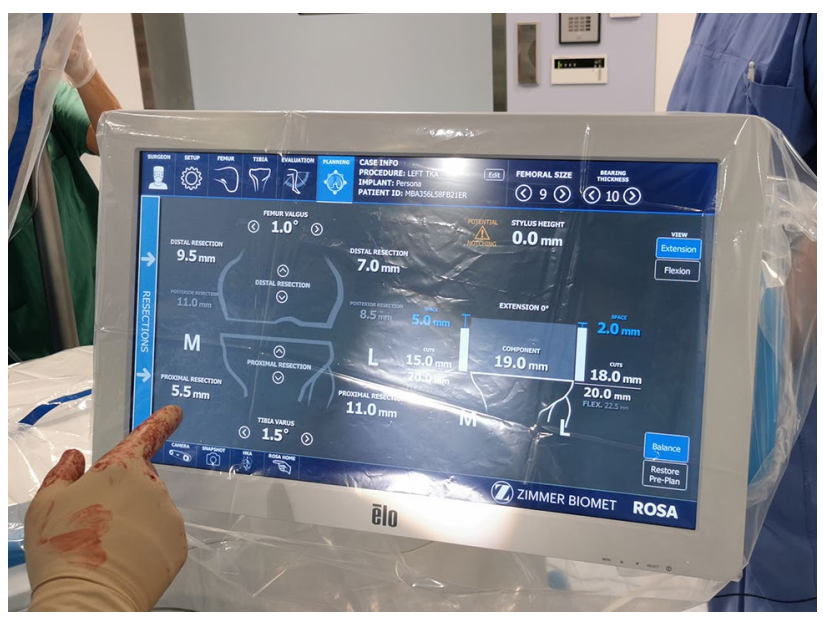

Fig. 1 Intraoperative planning: resections are calculated on the acquired bony landmarks and ligament assessment to obtain the desired balance and HKA axis 


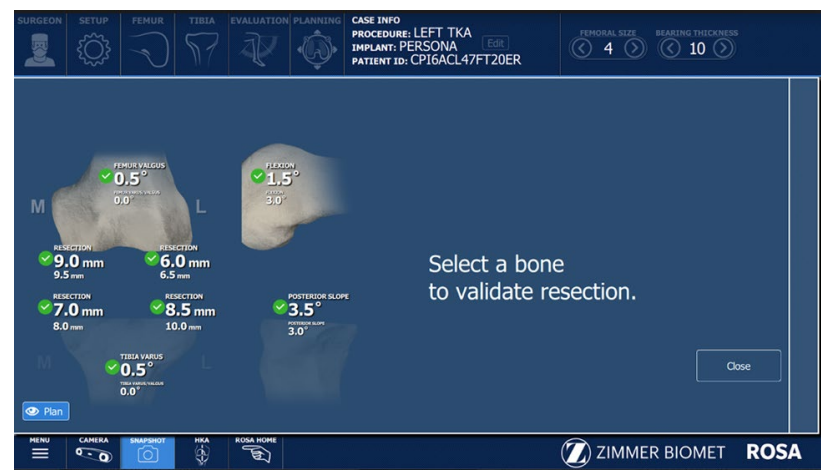

Fig. 2 Live validation system

by Biant [3]. For the evaluation of the reproducibility of HKA planned alignment the following X-ray assessment was conducted: preoperatively full-leg and lateral X-ray view obtained for each single patient. Postoperatively, full-leg weight bearing $\mathrm{X}$-rays were obtained for every patient on the second day after surgery and at 3 months follow up as a standard protocol in our institution. Radiographic measurements were done with the use of Carestream Vue PACS v11.3.4 (Carestream Health, Inc, Rochester, NY).

\section{Robotic procedure}

The robotic procedures were performed as described by Parratte in a previous publication [19].

In summary, the intraoperative planning is performed by the surgeon, using the dedicated software, to determine the ideal resection thickness and angles to obtain a balanced and well-aligned TKA (Fig. 1 shows the calculated resections once the desired extension gap balance is achieved). All the planned values are automatically recorded by the system. The planned measurements are entered into the ROSA system and verified perioperatively in situ during the surgery. The robotic arm positions and holds the universal cutting jig in the desired location as determined by the surgical plan.

Once the cutting jig is set in the correct position it is pinned to the bone and the surgeon performs the cuts. After femoral and tibia resections, the surgeon can verify and validate the cuts with a specific validation tool (Fig. 2) in this study the cuts were also measured and with a calliper. Once the first two cuts are performed, the extension gap evaluation can be performed and verified either with a static spacer or with a balancing tool (Zimmer Fuzion ${ }^{\circledR}$ spacer block or tensor). The flexion gap and the rotation of the femoral component are then re-assessed in the planning system with a rotational that is based on the ligament tension assessed with the Zimmer Fuzion ${ }^{\circledR}$ system (spacer block or tensioner). The robotic arm is used to position the drilling holes for the dedicated classic 4-in-1 posterior referenced cutting jig that sets the anterior-posterior position and rotation of the femoral component, according to the planning. The remaining femoral cuts are then performed. After the cuts are performed, posterior condyles cuts thickness is measured with a calliper and flexion knee balance is evaluated with the robot and Fuzion balancing tool. The preparation of the tibial metaphysis and the patella are performed with a standard instrumentation. Trial implants are then inserted, and the final axis of the leg is recorded and compared with the intra-operative plan.

The study didn't aim for a specific HKA; the HKA was intraoperatively planned to obtain balanced gaps, ranging $\pm 3^{\circ}$ from the MA (Mechanical Axis) in the majority of cases (in the present study in 1 case the final target was set at $+5^{\circ}$ from the MA); one of the aims of the study was to compare and validate the planned intra-op HKA and the post-op HKA.

\section{Statistical analysis}

To confirm the reliability of the measurements, interobserver, and intraobserver comparisons were performed on the pre- and post-op X-rays evaluation (HKA was measured in the pre-op and at the 3 months follow up X-rays evaluation). For each parameter studied (cuts and angles: planned with the ROSA system, measured with a dedicated Vernier calliper or calculated on the X-rays and validated with the ROSA system) descriptive statistics (mean and standard deviation) were calculated. For each paired samples the average error was calculated (mean of the differences between coupled data calculated for every patient) and a dedicated $t$ test was performed. A confidence interval of $95 \%$ was set a priori for the $t$ test. Statistical significance was set at $P<0.05$ for all statistical tests. All statistics were performed using Excel software (version 2016; Microsoft, Redmond, WA).

\section{Results}

Average results ( \pm standard deviation SD) of the planned, measured and validated cuts and angles together with their histogram are presented in Tables 1 and 2 respectively.

A comparison between the average measures of the planned and the validated (obtained with the validation tool of the system) data provided by the ROSA system (femoral and tibial cuts, femoral flexion and axis, tibial slope and axis) as well as between the planned, validated and measured cuts thicknesses (with the calliper) and angles (and on the post-operative X-rays) was performed and reported on Tables 3 and 4. 
Table 1 Average values of the cuts, planned (APC)—validated with ROSA system (AVC) measured with calliper (AMC) with histogram
Table 2 Average values of the angles, planned (APA) validated with ROSA system (AVA)-measured on the $\mathrm{x}$-rays (AMA) with histogram

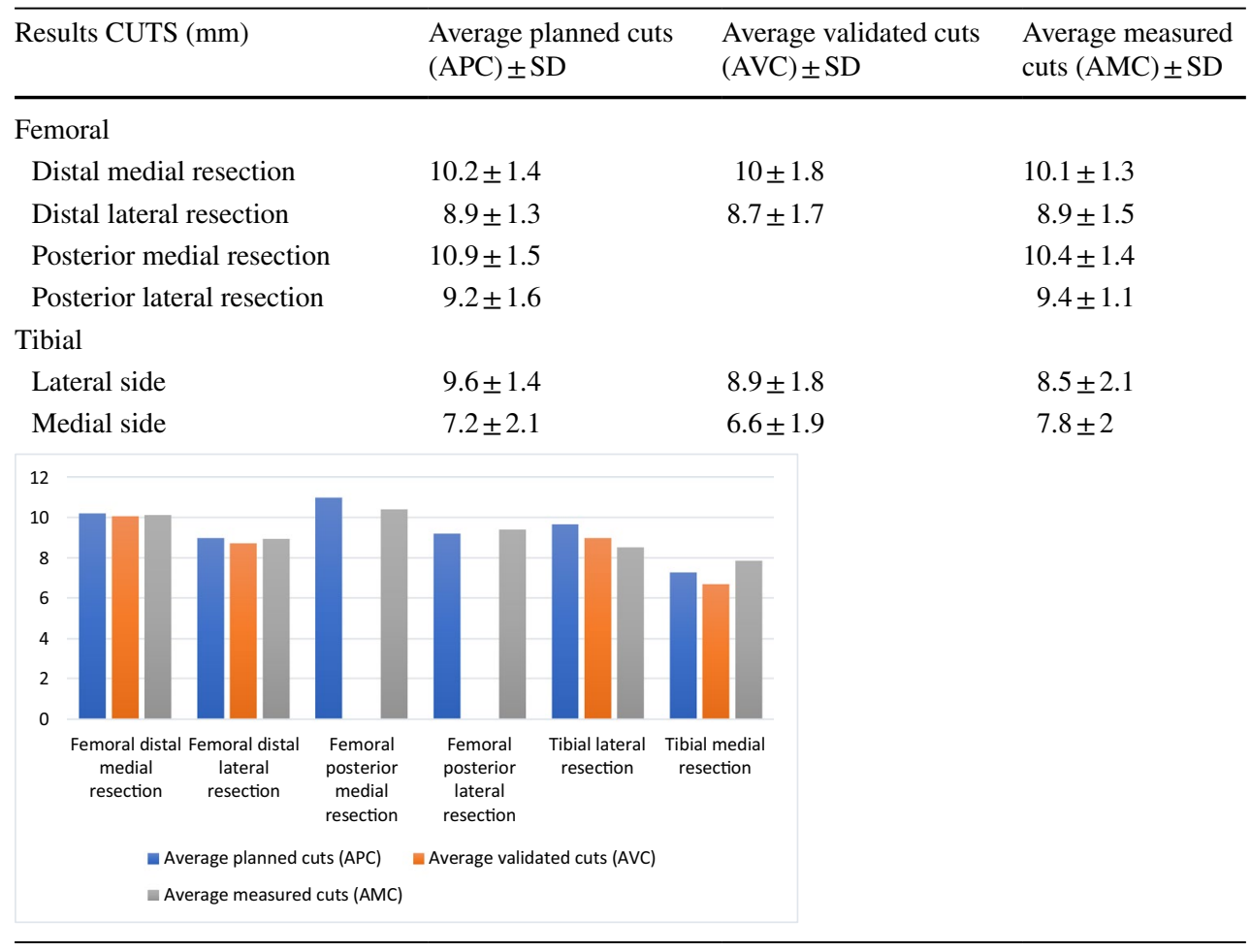

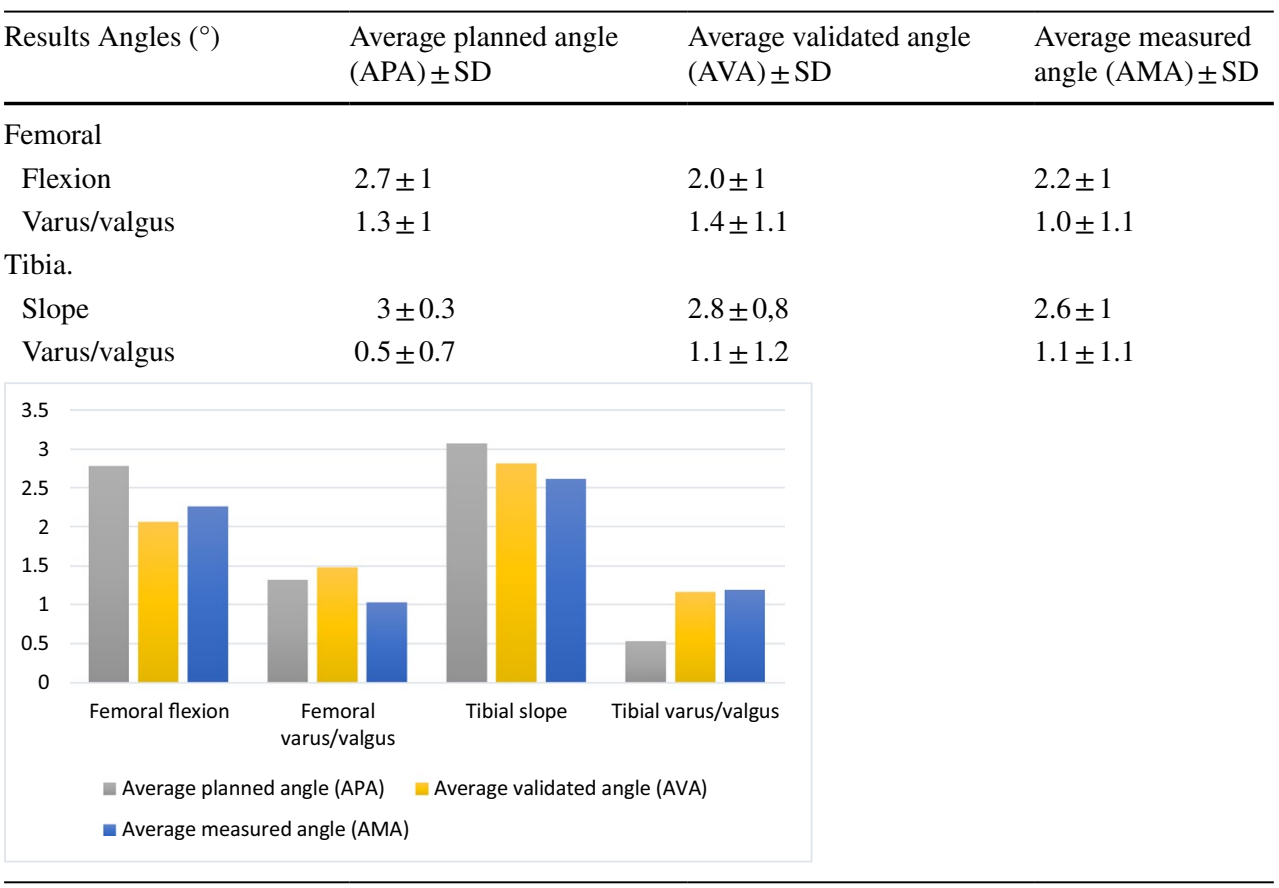

A statistically significant difference was found only between: the average planned and validated angle of the femoral flexion, the average planned and validated angle of the tibial coronal axis and the value of the planned and verified thickness of the medial and lateral tibial cuts.
The average difference was in any case below $1 \mathrm{~mm}$ or under 1 degree with $\mathrm{SD}<1$. No statistically significant difference for any of the other measures was found (Tables 3 and 4). Long standing weight bearing Postoperative X-rays, were performed at 3 months follow up as 
Table 3 Average difference (mean of the differences between the two measurements for each patient), and SD (standard deviation) with histogram between: average planned cuts (APC); average validated cuts (AVC) and average measured cuts (AMC)
Table 4 Average difference (mean of the differences between the two measurements for each patient), and SD (standard deviation) with histogram between: average planned angles (APA); average validated angles (AVA) and average measured angles (AMA)

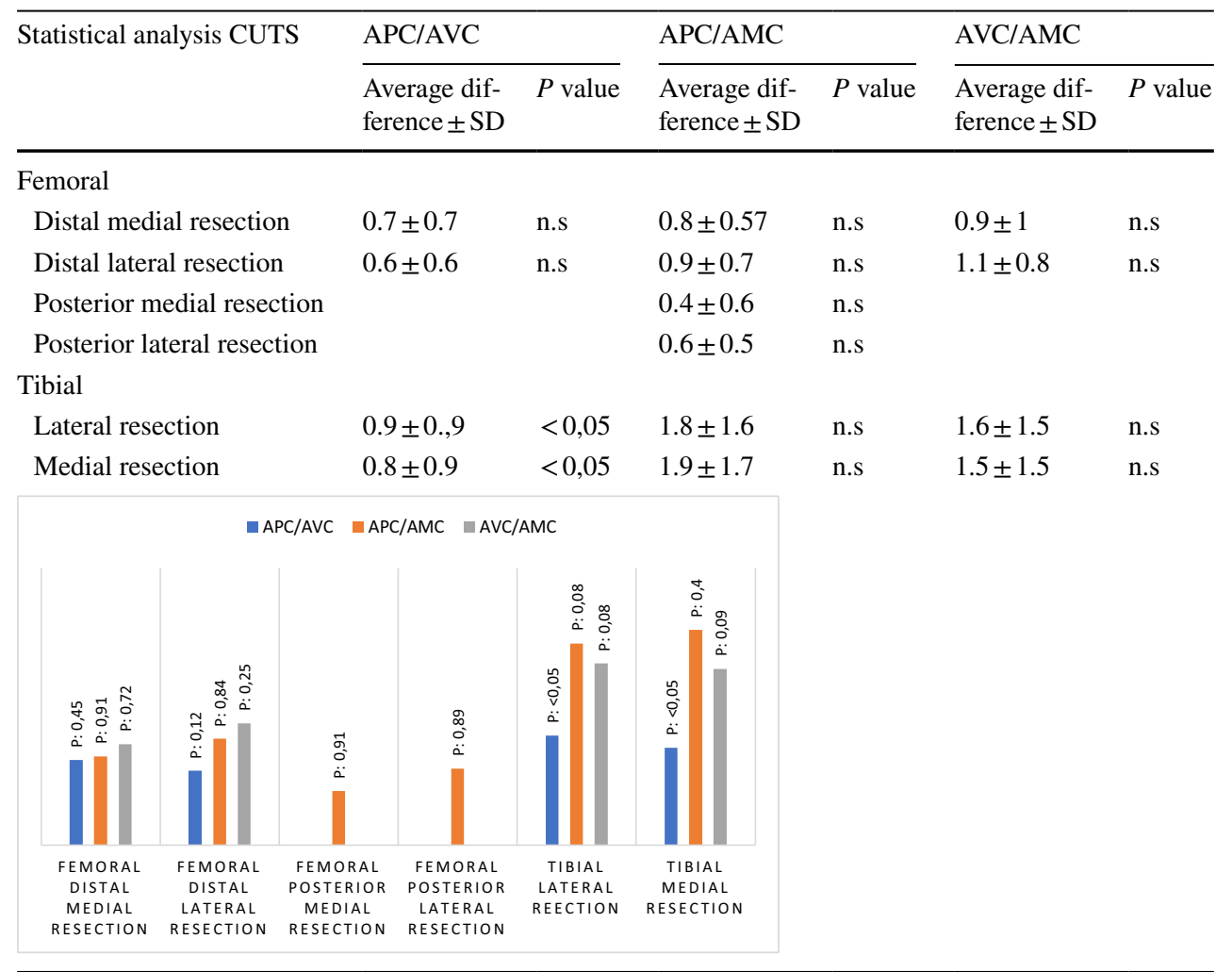

a standard protocol at our institution. The distribution of the post-op HKA alignment around the mechanical axis is represented in Fig. 3. The post-op coronal alignment was calculated and compared with the pre-op and the intra-op planned measures. The average difference between planned HKA and measured was $1.2 \pm 1.1$. No statistically significant difference was found (Table 5). 


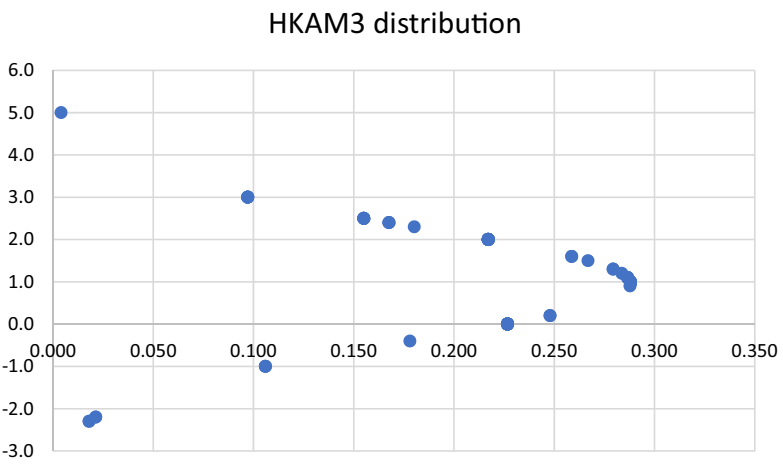

Fig. 3 Distribution of the HKA axis measured at the 3 months follow up X-rays

\section{Discussion}

This is a preliminary feasibility study. The primary hypothesis was that with the robotic assisted system in use in our institution (ROSA Zimmer-Biomet), based on a robotic assisted positioning of the cutting jig, a high level of accuracy of cuts, angular values and post-op limb alignment would be achieved.

The most important finding of the present study is that this new Robotic System demonstrated high accuracy in performing the planned cuts and angles in vivo.
First, the cuts, planned and verified with the robotic validation tool, available from the robots' surgical reports, were compared. The differences were less than $1 \mathrm{~mm}$ for both the femur and the tibia with a standard deviation of less than 1 . Using a traditional saw system for cutting the bone, this result is justifiable with the thickness of the blade itself. A statistically significant difference was found only between the planned and validated cuts of the tibia, but no statistically significant difference was registered between planned and measured or measured and validated cuts.

The difference between planned and obtained angles both for the femur (flexion and coronal axis) and the tibia (slope and coronal axis) was less than 1 degree with a standard deviation of less than 1 . A statistically significant difference was found only between the average planned and validated angle of the femoral flexion and of the tibial coronal axis and was in any case below 1 degree with a SD of less than 1 . This data can be justified by a manual error of the surgeon when applying the validation tool on the bone surface: the flat tool, applied on the bone surface have a small degree of freedom and the measure can be influenced by the rough surface of the bone.

The reliability of the robot in reproducing the planned HKA was verified with the axis measured on long length $\mathrm{x}$-rays at the 3 months follow up: the mean difference was $1.2 \pm 1.1$. No statistically significant difference was found.

Table 5 Planned and measured HKA axes on long standing X-rays and average difference (in degrees) between planned and post-op measured HKA (mean of the differences between the HKA measurements for each patient) and SD (standard deviation), with histogram

\begin{tabular}{llll}
\hline Results HKA $\left(^{\circ}\right)$ & HKA preop & HKAP & HKAM3 \\
\hline Average & 175.8 & 178.2 & 179.0 \\
SD & 8.6 & 1.6 & 1.3 \\
\hline
\end{tabular}

Statistical analysis HKA

HKAP/HKAM3

$P$ value

Average difference \pm SD

n.s

$1.2 \pm 1.1$

Results HKA

Results $\left({ }^{\circ}\right)$ : HKA preop; HKAP: HKA planned; HKAM3: HKA measured 3 months.

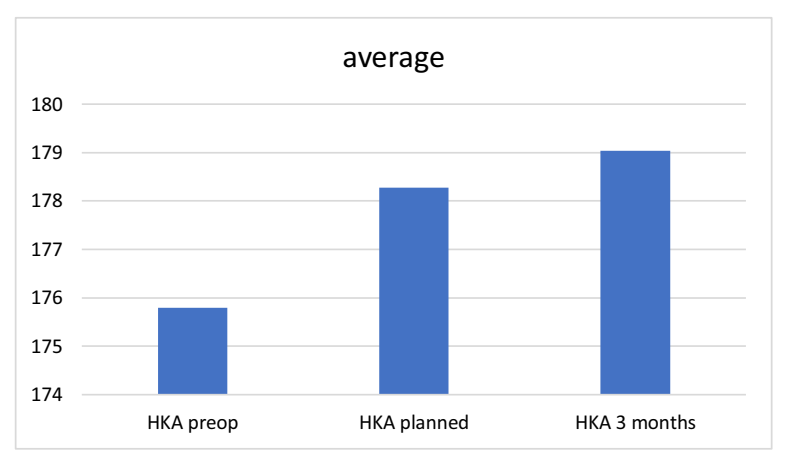


All These findings are relevant to support the reliability of the robotic system.

Most of the recent studies presented in the literature on robotic surgery focus on alignment of the components and post-operative HKA axis [16, 22, 23].

Aiming to a specific degree of alignment requires precise systems to study the preoperative conditions and obtain the planned axis, and with these targets an accurate robotic system may be very helpful. In this study, the average error was $1.2 \pm 1.1^{\circ}$.

The results of the present study confirm, in vivo, those obtained and validated by Parratte et al. [19] in their cadaveric study where they performed 30 TKA on 15 frozen cadaveric specimens with the ROSA Knee System (Zimmer Biomet) using 3 different implants: the femoral and tibial coronal and sagittal alignment error showed a standard deviation of less than $1(\mathrm{SD}<1)$, measured with the Optical Navigation System (ORTHOsoft, Zimmer Biomet, IN). The authors also described a difference between planned and measured bone resections (as well with a calliper) with a $\mathrm{SD}<1$, which is again a result similar to the one obtained in vivo in the present study.

Finally, they described a mean hip-knee-ankle axis difference of $0.03 \pm 0.87$ between the measured and the planned axis, with a rate of malalignment $>3^{\circ}$ of $0 \%$.

This study is also in line with the one presented by Figueroa et al. [8]who focused on the reliability in reproducing the planned cuts with a robotic-arm system that incorporates robotic placement of the cutting jigs. In their study Figueroa et al. [8] presented 173 TKA performed with an imageless passive robotic TKA. They compared intraoperative recorded cuts with postoperative CT scans for each case. They described a mean difference for HKA predicted and measured of $0.4 \pm 2.4^{\circ}$ more varus, with $83 \%$ HKA angles within a difference of 3 degrees.

In a recent publication, Collins [7] showed results comparable to those presented in this study using the Navio Robotic System (Smith and Nephew) with a 93\% accuracy on the coronal alignment. In their study Sires et al. present the results using the Mako total knee robotic arm-assisted surgery (Stryker, Kalamazoo, MI) in 37 consecutive cases of TKA; they measured angular and thickness average difference between the planning and the results obtained with a navigated probe system. They described for the femoral component a mean absolute difference from the plan of $0.38 \mathrm{~mm}$ for the distal femoral cut, of $0.46^{\circ}$ for varus/valgus and of $0.55^{\circ}$ for flexion. For the tibial cuts the mean absolute difference was $0.37 \mathrm{~mm}$, with $0.53^{\circ}$ for valgus/varus and $0.59^{\circ}$ for the anterior/posterior slope.

Hamp et al. [11] also showed greater accuracy with a Robotic Arm Assisted TKA (MAKO, Stryker, Kalamazoo, MI) comparing to a traditional technique in an in-vitro study on 12 knees, and recently Held [12] showed improved compartment balancing using a Robotic assisted system (Navio, Smith and Nephew) for total knee arthroplasty.

The results of the present study are also in line with those presented by these ones.

In a recent systematic review and meta-analysis, Zhang [26] showed that Robotic TKA (RATKA) demonstrated improved accuracy of component positioning and patientreported outcomes with a very short learning curve for operating time (between 7 and 11 cases), comparing to traditional techniques.

Same results were previously showed by Kayani [14]that demonstrated improved accuracy in bone cuts and implant positioning with a robotic arm assisted TKR, a short learning curve of 7 cases, and no effect on the accuracy of implant positioning.

Moreover, two recent studies show an improved accuracy in implant positioning using a robotic system, although only the meta-analyses published by Agarwal [1] outlines an associated improvement on patient's outcomes. In fact, the data published in the overview performed by Kort [15] show concerns and inconclusive results in terms of improvements of clinical scores or reduction of complications and revisions. On the other side, in a recent publication Yamamura [24] showed only fair-to-moderate accuracy in terms of bone cuts using a CT-based Patients Specific Instrumentation (PSI) system, confirming the disappointing results with PSI, in terms of accuracy, previously presented by An et al. [2] with an MRI-based system. Zambianchi also showed low accuracy of two different PSI systems [25].

All these studies showed that the limitations in accuracy of PSI systems were mainly related to the positioning of the cutting jigs despite a correct planning [2, 24, 25]. The use of a Robotic Arm may be helpful to overcome these limitations improving the positioning of the cutting jig and the accuracy of the bone cuts.

This study has several limitations: first, the absence of any clinical evaluation of the patients, but the aim of the study was to validate the in vivo the accuracy of the system in terms of bone resections and alignment, and not to compare these data with any clinical evaluation. Second limitation is the fact that all procedures were imageless even if the system have an image-based option. The reasons for this choice are mainly two: first, the study have been conducted during the first and second wave of covid-19 outbreak and patients had limited access to hospitals, so it was logistically complicated and risky to organize the pre-op study for the image-based planning, second because once completed the learning curve with the image-less technique we decided to validate this technique before starting a new learning curve.

On the other hand, this last limitation results in a strength as the high level of accuracy demonstrated by the imageless procedure implies different logistic and economic advantages such as a reduced number of hospital access for the 
patients, no dedicated pre-op x-ray examination and no waiting time for the approved pre-op planning by the company.

This, it is, to our knowledge, the first study validating the accuracy of this specific robotic system in vivo.

More research is needed to assess whether the improvement of accuracy correlates with better results in patient satisfaction and overall survival rates of total knee replacements.

\section{Conclusions}

The results of this study demonstrate that a high degree of accuracy can be obtained using this new robotically assisted TKA system based on robotically positioned and stabilized cutting jigs.

Funding No funding were received for the present study.

\section{Declarations}

Conflict of interest Prof Francesco Benazzo declares a teaching contract with the manufacturer (Zimmer Biomet); all other authors declare no conflict of interest related to the present study.

Ethical approval No IRB approval was necessary for this study, as a standard and approved technique was applied and no sensible data are presented.

Informed consent All patients signed an informed consent for the surgical procedure.

\section{References}

1. Agarwal N, To K, McDonnell S, Khan W (2020) Clinical and radiological outcomes in robotic-assisted total knee arthroplasty: a systematic review and meta-analysis. J Arthroplast 35:3393-3409

2. An VVG, Sivakumar BS, Phan K, Levy YD, Bruce WJM (2017) Accuracy of MRI-based vs. CT-based patient-specific instrumentation in total knee arthroplasty: a meta-analysis. J Orthop Sci 22:116-120

3. Biant LC, Yeoh K, Walker PM, Bruce WJM, Walsh WR (2008) The accuracy of bone resections made during computer navigated total knee replacement. Do we resect what the computer plans we resect? Knee 15:238-241

4. Canovas F, Dagneaux L (2018) Quality of life after total knee arthroplasty. Orthop Traumatol. https://doi.org/10.1016/j.otsr. 2017.04.017

5. Chen AF, Kazarian GS, Jessop GW, Makhdom A (2018) Current concepts review: robotic technology in orthopaedic surgery. J Bone Joint Surg 100:1984-1992

6. Cidambi KR, Robertson N, Borges C, Nassif NA, Barnett SL (2018) Intraoperative comparison of measured resection and gap balancing using a force sensor: a prospective, randomized controlled trial. J Arthroplast 33:S126-S130

7. Collins K, Agius PA, Fraval A, Petterwood J (2021) Initial experience with the navio robotic-assisted total knee replacement-coronal alignment accuracy and the learning curve. J Knee Surg. https://doi.org/10.1055/s-0040-1722693

8. Figueroa F, Wakelin E, Twiggs J, Fritsch B (2019) Comparison between navigated reported position and postoperative computed tomography to evaluate accuracy in a robotic navigation system in total knee arthroplasty. Knee 26:869-875

9. Gunaratne R, Pratt DN, Banda J, Fick DP, Khan RJK, Robertson BW (2017) Patient dissatisfaction following total knee arthroplasty: a systematic review of the literature. J Arthroplast 32:3854-3860

10. Scott HCE, Turnbull GS, MacDonald D, Breusch SJ, Joint BJ (2017) Activity levels and return to work following total knee arthroplasty in patients under 65 years of age. Bone Joint J 99:1037

11. Hampp EL, Chughtai M, Scholl LY, Sodhi N, Bhowmik-Stoker M, Jacofsky DJ, Mont MA (2019) Robotic-arm assisted total knee arthroplasty demonstrated greater accuracy and precision to plan compared with manual techniques. J Knee Surg 32:239-250

12. Held MB, Grosso MJ, Gazgalis A, Sarpong NO, Boddapati V, Neuwirth A, Geller JA (2021) Improved compartment balancing using a robot-assisted total knee arthroplasty. Arthroplast Today $7: 130-134$

13. Kayani B, Konan S, Ayuob A, Onochie E, Al-Jabri T, Haddad FS (2019) Robotic technology in total knee arthroplasty: a systematic review. EFORT Open Rev 4:611-617

14. Kayani B, Konan S, Huq SS, Tahmassebi J, Haddad FS (2019) Robotic-arm assisted total knee arthroplasty has a learning curve of seven cases for integration into the surgical workflow but no learning curve effect for accuracy of implant positioning. Knee Surg Sports Traumatol Arthrosc 27:1132-1141

15. Kort N, Stirling P, Pilot P, Müller JH (2021) Robot-assisted knee arthroplasty improves component positioning and alignment, but results are inconclusive on whether it improves clinical scores or reduces complications and revisions: a systematic overview of meta-analyses. Knee Surg Sports Traumatol Arthrosc. https://doi. org/10.1007/s00167-021-06472-4

16. Liow MHL, Xia Z, Wong MK, Tay KJ, Yeo SJ, Chin PL (2014) Robot-assisted total knee arthroplasty accurately restores the joint line and mechanical axis: a prospective randomised study. J Arthroplast 29:2373-2377

17. Mercuri JJ, Pepper AM, Werner JA, Vigdorchik JM (2019) Gap balancing, measured resection, and kinematic alignment: how, when, and why? JBJS Rev. https://doi.org/10.2106/jbjs.rvw.18.00026

18. Pang HN, Yeo SJ, Chong HC, Chin PL, Ong J, Lo NN (2011) Computer-assisted gap balancing technique improves outcome in total knee arthroplasty, compared with conventional measured resection technique. Knee Surg Sports Traumatol Arthrosc 19:1496-1503

19. Parratte S, Price AJ, Jeys LM, Jackson WF, Clarke HD (2019) Accuracy of a new robotically assisted technique for total knee arthroplasty: a cadaveric study. J Arthroplast 34:2799-2803

20. Rosso F, Cottino U, Dettoni F, Bruzzone M, Bonasia DE, Rossi R (2019) Revision total knee arthroplasty (TKA): Mid-term outcomes and bone loss/quality evaluation and treatment. J Orthop Surg Res $14: 280$

21. Savov P, Ettinger M, Tuecking L-R (2020) PSI-technique for kinematic alignment. Orthopade. https://doi.org/10.1007/ s00132-020-03927-3

22. Song EK, Seon JK, Yim JH, Netravali NA, Bargar WL (2013) Robotic-assisted TKA reduces postoperative alignment outliers and improves gap balance compared to conventional TKA knee. Springer, New York, pp 118-126

23. Vaidya NV, Deshpande AN, Panjwani T, Patil R, Jaysingani T, Patil P (2020) Robotic-assisted TKA leads to a better prosthesis alignment and a better joint line restoration as compared to conventional TKA: a prospective randomized controlled trial. Knee Surg Sports Traumatol Arthrosc. https://doi.org/10.1007/s00167-020-06353-2 
24. Yamamura K, Inori F, Konishi S (2021) Evaluation of the accuracy of resected bone thickness based on patient-specific instrumentation during total knee arthroplasty. Arch Orthop Trauma Surg 141:1583-1590

25. Zambianchi F, Colombelli A, Digennaro V, Marcovigi A, Mugnai R, Fiacchi F, Sandoni D, Belluati A, Catani F (2017) Assessment of patient-specific instrumentation precision through bone resection measurements. Knee Surg Sports Traumatol Arthrosc 25:2841-2848

26. Zhang J, Ndou WS, Ng N, Gaston P, Simpson PM, Macpherson GJ, Patton JT, Clement ND (2021) Robotic-arm assisted total knee arthroplasty is associated with improved accuracy and patient reported outcomes: a systematic review and meta-analysis. Knee Surg Sports Traumatol Arthrosc. https://doi.org/10.1007/ s00167-021-06464-4

Publisher's Note Springer Nature remains neutral with regard to jurisdictional claims in published maps and institutional affiliations. 